\title{
Adsorption of Toxic Heavy Metal Methylmercury (MeHg) on Germanene in Aqueous Environment: A First-Principles Study
}

\author{
Muhammad Rifqi Al Fauzan ${ }^{1}$, Trias Prima Satya ${ }^{1}$, Galih Setyawan ${ }^{1}$, Imam Fahrurrozi $^{1}$, Fitri \\ Puspasari $^{1}$, Juliasih Partini ${ }^{2}$, and Sholihun Sholihun ${ }^{2^{*}}$ \\ ${ }^{1}$ Department of Electrical Engineering and Informatics, Vocational College, Universitas Gadjah Mada, \\ Sekip Unit III, Yogyakarta 55281, Indonesia \\ ${ }^{2}$ Department of Physics, Faculty of Mathematics and Natural Sciences, Universitas Gadjah Mada, \\ Sekip Utara BLS 21, Yogyakarta 55281, Indonesia
}

\section{*Corresponding author:}

email:sholihun@ugm.ac.id

Received: June 22, 2021

Accepted: August 27, 2021

DOI: $10.22146 /$ ijc. 66902

\begin{abstract}
We perform first-principles calculations to investigate the adsorption process of methyl mercury ( $\mathrm{MeHg}$ ) on germanene with the presence of water molecules. We calculate the formation energy and density of states to determine the effect of the adsorption of $\mathrm{MeHg}$ on the structural and electronic properties of germanene. Our results show that $\mathrm{MeHg}$ is chemisorbed on germanene through a spontaneous reaction. The calculated formation energy of the system is $-1.61 \mathrm{eV}$. We also carry out charge distribution and charge transfer calculations based on the Mulliken model to understand the adsorption mechanism of $\mathrm{MeHg}$.
\end{abstract}

Keywords: methylmercury; germanene; adsorption; formation energy; the density of states

\section{- INTRODUCTION}

Mercury ( $\mathrm{Hg}$ ) poses a severe danger to humans and the environment, e.g., $\mathrm{Hg}$ exposure causes a wide range of health problems. This element can affect the lungs, brain, and skin and cause permanent damage to the nervous system [1-2]. Anthropogenic and natural sources contribute to the accumulation of $\mathrm{Hg}$ in soil. With intervention from microbial methylation reaction, inorganic $\mathrm{Hg}$ in the soil can be transformed into methylmercury (MeHg), one of the most toxic forms of organic Hg [3]. The MeHg molecule penetrates from soil to aquatic systems through runoff. It can bioaccumulate and biomagnify up the aquatic food chain. Therefore, $\mathrm{MeHg}$ is a major hazard to humans [4].

Remediation techniques are needed to control $\mathrm{MeHg}$ contamination in aquatic systems. Wang et al. reported several techniques that are needed to counter the MeHg pollution [5]. They explained the commonly used and emerging remediation techniques such as stabilization (solidification), nanotechnology, phytoextraction, etc. This study uses the adsorption mechanism by using germanene, as a host material. Germanene is a two- dimensional germanium-based material analog of graphene [6] and has been successfully grown on various substrates such as $\mathrm{Au}(111)$ [7], $\mathrm{Pt}(111)$ [8], and $\mathrm{Al}(111)$ [9]. Germanene exhibits strong sensitivity to chemical and environmental changes. Previous studies showed that germanene successfully adsorbs $3 \mathrm{~d}$ transitional metal atoms (Sc, Ti, V, Cr, Mn, Fe, Co, Ni, Cu, and Zn) [10], alkali metal atoms ( $\mathrm{Li}, \mathrm{Na}$, and $\mathrm{K}$ ) [11-12] and various gas molecules [13]. From the calculated adsorption energy, germanene more easily adsorbs small gas molecules than silicene [14-15]. Therefore, the study of molecule adsorption on germanene, particularly that of a toxic molecule such as MeHg is necessary.

In the present work, we perform first-principles density-functional-theory (DFT) calculations to investigate the capability of germanene to adsorb the heavy metal $\mathrm{MeHg}$ in aqueous conditions. Supercell with 32 atomic sites is involved, and all atoms are relaxed to find the optimized geometry. We calculate the formation energy ( $\left.\mathrm{E}_{\text {form }}\right)$, the density of states (DOS), and charge transfer to study the adsorption mechanism of MeHg in germanene. 


\section{- COMPUTATIONAL METHODS}

The DFT calculations are performed using OpenMX (Open-source package for Material eXplorer) code [1618] based on pseudo-atomic localized basis functions. In this code, the norm-conserving pseudopotential is used [19], and the wave functions are expanded by a linear combination of multiple pseudo-atomic orbitals [17-18]. The exchange-correlation functional is evaluated using the generalized gradient approximation with the PerdewBurke-Ernzerhof method [20]. A 25-point grid is applied in the Brillouin zone integration. The energy convergence of the self-consistent calculations is adjusted at $10^{-4} \mathrm{Ha}$.

In this study, we use a supercell model in which the germanene surface lies on the $x-y$ plane (Fig. 1). The supercell is generated by using a two-atoms unit cell with the optimized lattice constant. Lattice parameter optimization is carried out using the Murnaghan equation of states as follows [21-26]:

$\mathrm{E}(\mathrm{V})=\mathrm{E}_{0}+\mathrm{B}_{0} \mathrm{~V}_{0}\left[\frac{1}{\mathrm{~B}_{0}^{\prime}\left(\mathrm{B}_{0}^{\prime}-1\right)}\left(\frac{\mathrm{V}}{\mathrm{V}_{0}}\right)^{1-\mathrm{B}_{0}^{\prime}}+\frac{1}{\mathrm{~B}_{0}^{\prime}}\left(\frac{\mathrm{V}}{\mathrm{V}_{0}}\right)-\frac{1}{\mathrm{~B}_{0}^{\prime}-1}\right]$

where $\mathrm{E}_{0}, \mathrm{~V}_{0}, \mathrm{~B}_{0}$, and $\mathrm{B}_{0}^{\prime}$ are the fitting parameters calculated at the constant volume. $\mathrm{E}_{0}$ is total energy, and $\mathrm{V}_{0}$ is lattice volume while $\mathrm{B}_{0}$ and $\mathrm{B}_{0}^{\prime}$ are bulk modulus and its first derivative, respectively.

A $20 \AA$ vacuum parameter, which is large enough to prevent interactions between adjacent layers, is applied perpendicularly to the germanene surface (Fig. 1(b)). A $4 \times 4 \times 1$ supercell containing 32 atoms is used as the host material. To model the aqueous environment, we introduce water molecules on the surface of germanene. We then calculate the formation energy $\mathrm{E}_{\text {form }}$ and charge transfer. The $E_{\text {form }}$ is defined by using the following equation [27-31]:

$\mathrm{E}_{\text {form }}=\mathrm{E}_{\text {subs/host }}-\left(\mathrm{E}_{\text {subs }}+\mathrm{E}_{\text {host }}\right)$ where $E_{\text {subs, }} E_{\text {host }}$, and $E_{\text {subs/host }}$ are the total energies of the isolated substrate, clean host material, and adsorption system, respectively. The same size of the unit cell is used for the system of interest. Using this definition, a negative value of $\mathrm{E}_{\text {form }}$ indicates spontaneous adsorption, and a positive value refers to non-spontaneous adsorption where the adsorbed state has more positive energy than the isolated one. Thus, external energy is needed to carry out the adsorption process. Meanwhile, the charge density difference was obtained by subtracting the total charge of the adsorption system from the individual charges of the isolated substrate and the host material [14,31], as follows:

$\Delta \rho=\rho_{\mathrm{MeHg} / \mathrm{Ge}}-\left(\rho_{\mathrm{MeHg}}+\rho_{\mathrm{Ge}}\right)$

where $\rho_{\mathrm{MeHg}}, \rho_{\mathrm{Ge}}$, and $\rho_{\mathrm{MeHg} / \mathrm{Ge}}$ are the charge density of $\mathrm{MeHg}$ with water molecules, germanene, and the adsorption system, respectively.

\section{- RESULTS AND DISCUSSION}

\section{Germanene in an Aqueous Environment}

We perform first-principles calculations of the germanene supercell with the presence of water molecules and $\mathrm{MeHg}$ molecule. We first optimize the

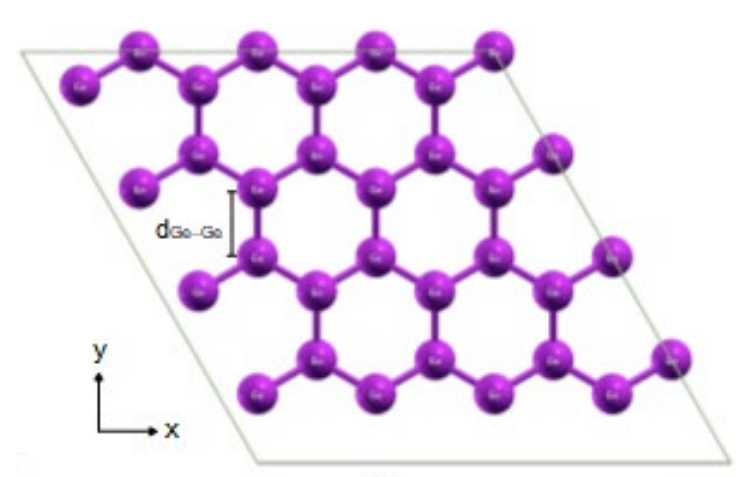

(a)

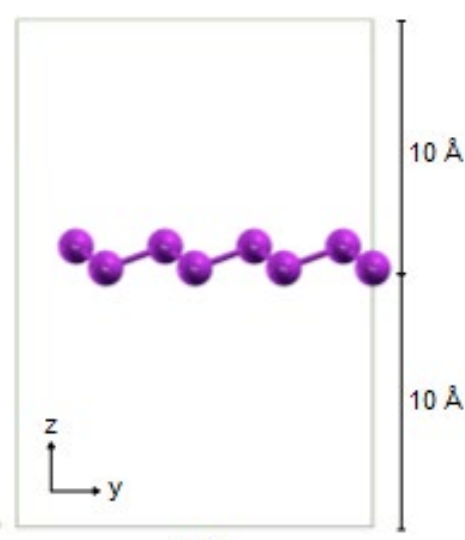

(b)

Fig 1. Pristine germanene supercell: (a) front-view overlaying the $x-y$ plane and (b) side-view showing the vacuum parameter in the z-direction. The purple balls denote germanium atoms 
germanene unit cell. The lattice constant of $4.06 \AA$ is calculated using Eq. (1). This result agrees with the previous calculations $[6,25]$. The lattice constant of germanene is higher than that of silicene (3.86 $\AA$ ) [32]. As for the bond lengths, the calculated $\mathrm{Ge}-\mathrm{Ge}$ bond (d) is $2.46 \AA$, which is slightly higher than that of the Si-Si bond in silicene (2.24 $\AA$ ) [33]. This result is in agreement with the results of previous theoretical [25,34] and experimental studies [7].

We next calculate the formation energy $\mathrm{E}_{\text {form }}$ using Eq. (2) and found that the system of germanene with water molecules $\left(\mathrm{H}_{2} \mathrm{Os} / \mathrm{G}\right)$ has $\mathrm{E}_{\text {form }}$ of $+0.05 \mathrm{eV}$. The positive value of $E_{\text {form }}$ implies the endothermicity of the adsorption process. Therefore, the adsorption only occurs in the presence of external energy.

We calculate the DOS to determine the electronic properties of the systems. Our results show that the presence of water molecules on the surface of germanene causes no change in the electronic state near the Fermi level, i.e., the shape of the DOS is the same, and there is no gap created. As shown in Fig. 2(a), the electronic state of pristine germanene is a semimetal with a zero bandgap at Dirac point. With the presence of water, the electronic state remains the same as in the pristine germanene (Fig. 2(b)). The difference in magnitude of the DOS at some energy ranges, e.g., at about $-5 \mathrm{eV}$ and $+8 \mathrm{eV}$, is due to water molecules in the system.

\section{Adsorption of MeHg on germanene}

We introduce the $\mathrm{MeHg}$ molecule on germanene with water molecules, that is, MeHg/G. Fig. 3 shows the stable structure of this system. The calculated $E_{\text {form }}$ of
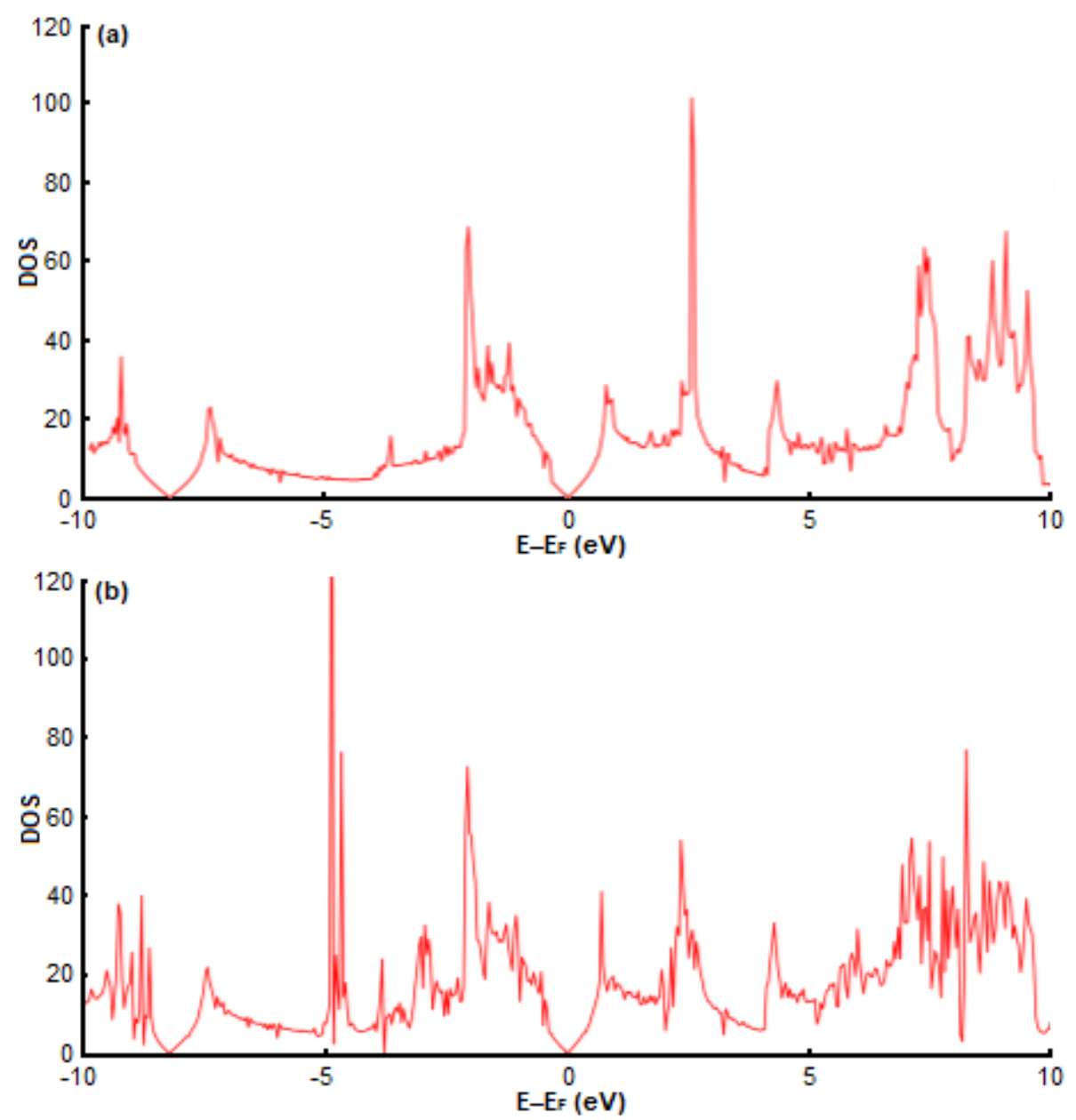

Fig 2. The density of states (DOS) of (a) pristine germanene and (b) germanene with water molecules. The Fermi level is set to zero 


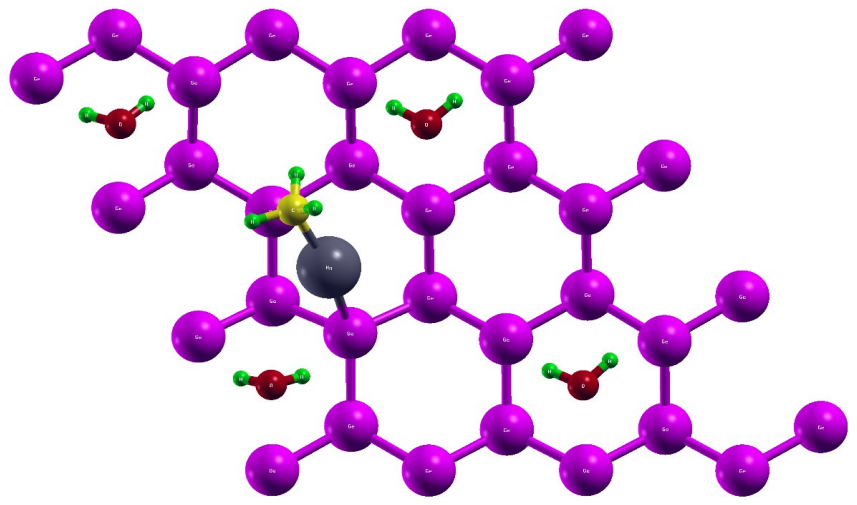

Fig 3. Stable structure of $\mathrm{MeHg}$ adsorption on germanene in aqueous condition. The black, yellow, green, red, and purple balls denote mercury, carbon, hydrogen, oxygen, and germanium atoms, respectively

$\mathrm{MeHg} / \mathrm{G}$ is $-1.61 \mathrm{eV}$. In contrast to the $\mathrm{H}_{2} \mathrm{Os} / \mathrm{G}$ system, the interaction between $\mathrm{MeHg}$ and germanene indicates a spontaneous reaction (negative $\mathrm{E}_{\text {form}}$ ). Therefore, the adsorption of $\mathrm{MeHg}$ on germanene easily occurs without external energy.

Calculations for electronic properties showed that the DOS of germanene changes after adsorbing $\mathrm{MeHg}$ (Fig. 4). The $\mathrm{MeHg}$ adsorption opens a narrow bandgap, making germanene a semiconductor with a $0.20 \mathrm{eV}$ bandgap. Ye et al. reported a similar result: germanene has a narrow bandgap ranging from $0.02 \mathrm{eV}$ to $0.31 \mathrm{eV}$ after adsorbing several alkali metal atoms [12].

The adsorption mechanism can be investigated by considering charge transfer between atoms in $\mathrm{MeHg}$ and germanium. We calculate the charge difference distribution $\Delta \rho$ as expressed in Eq. (3) to investigate the presence of charge transfer between two materials. Fig. 5 shows the isosurface of the charge and indicates the charge transfer between germanene and $\mathrm{MeHg}$ in an aqueous environment.

We then employ the Mulliken charge analysis to evaluate the net charge transfer between the materials. We observe that germanene loses its electron charge by about $0.25 \mathrm{e}$. On the other hand, heavy metal $\mathrm{MeHg}$ in an aqueous environment receives electron charge from germanene. This result agrees with the charge difference distribution as plotted in Fig. 5, where the depletion and accumulation of charges are observed between $\mathrm{MeHg}$ and $\mathrm{Ge}$ atoms of germanene.

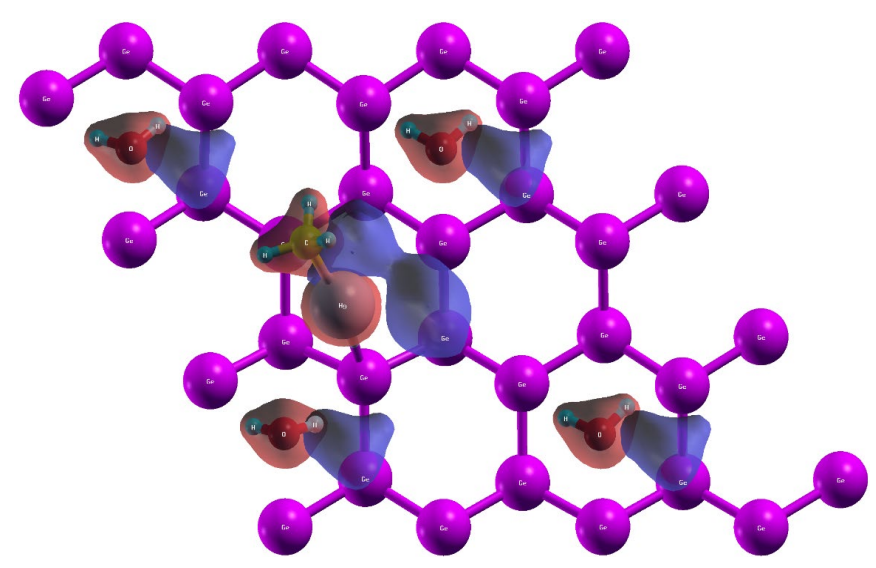

Fig 5. Charge difference distribution of the $\mathrm{MeHg} / \mathrm{G}$ system. Red and blue areas illustrate the accumulation and loss of charge, respectively. Electron charges move from blue to red areas

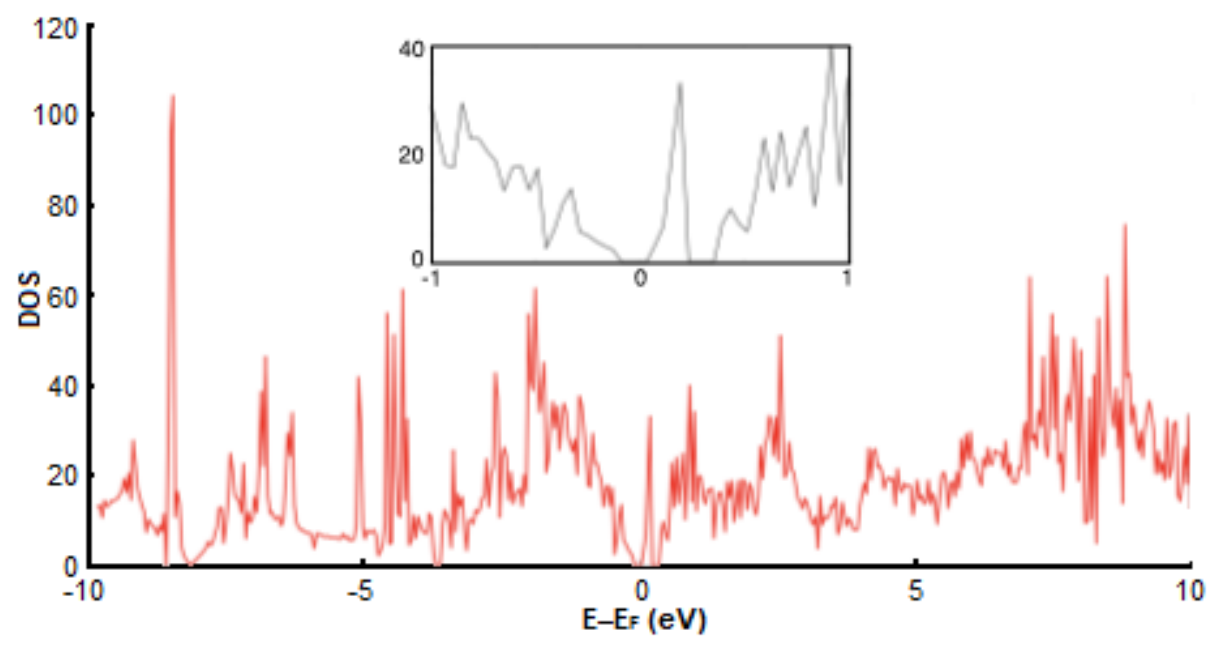

Fig 4. DOS of $\mathrm{MeHg} / \mathrm{G}$ system with magnification around the Fermi level 


\section{Atomic Path}

At the initial geometry, the $\mathrm{Hg}$ atom is placed on the center of the honeycomb structure of germanene with a vertical distance of $2.5 \AA$ to the surface of germanene (Fig. 6). During relaxation, the $\mathrm{Hg}$ atom diffuses to the $\mathrm{Gel}$ atom and creates a covalent bond. The calculated bond length of $\mathrm{Hg}-\mathrm{Ge} 1$ is $2.84 \AA$ in the transition geometry, whereas the bond length shortens to $2.70 \AA$ in the final geometry. The presence of $\mathrm{MeHg}$ affects the position of its adjacent atoms particularly Ge1-Ge13. As a result, the atoms undergo vertical distortions in various magnitudes, as listed in Table 1.

In the final geometry, the formation of $\mathrm{MeHg}$ remains as that of its molecular form with the $\mathrm{Hg}-\mathrm{C}$ and $\mathrm{C}-\mathrm{H}$ bond lengths of 2.19 and $1.11 \AA$ A respectively. Given that $\mathrm{MeHg}$ is symmetric under $\mathrm{C}_{3}$ (rotation by $2 \pi$ ) and symmetric under the vertical mirror $\sigma_{v}$, it shows a $C_{3 v}$ symmetry. Meanwhile, the water has a $\mathrm{C}_{2 \mathrm{v}}$ molecule with the O-H bond length of $0.99 \AA$.

\section{- CONCLUSION}

We perform DFT calculations to investigate the adsorption mechanism of $\mathrm{MeHg}$ on germanene with the presence of water molecules. $\mathrm{MeHg}$ is chemisorbed on germanene with a $\mathrm{E}_{\text {form }}$ of $-1.61 \mathrm{eV}$. To determine the mechanism of interaction, we carry out charge difference distribution and charge transfer calculations. Our results show that germanene distributes an electron charge of 0.25 e to $\mathrm{MeHg}$ under aqueous conditions.

\section{- ACKNOWLEDGMENTS}

The authors would like to thank Universitas Gadjah Mada for the computer facilities during finishing this research. In addition, the authors (S. and J.P.) thank the Ministry of Research and Technology for the financial support through the Penelitian Dasar 2021 research grant.

Table 1. Magnitudes of vertical distortion of $\mathrm{MeHg}$ neighboring atoms. The values are obtained by subtracting the initial state coordinates from the final state coordinates. Positive and negative values indicate that the Ge atom is moving away and moving closer to $\mathrm{MeHg}$ in the z-direction, respectively

\begin{tabular}{cccc}
\hline Atom & Magnitude $(\AA)$ & Atom & Magnitude $(\AA)$ \\
\hline Ge1 & 0.23 & Ge8 & 0.22 \\
Ge2 & 0.40 & Ge9 & 0.17 \\
Ge3 & 0.52 & Ge10 & -0.03 \\
Ge4 & 0.60 & Ge11 & 0.11 \\
Ge5 & 0.98 & Ge12 & 0.15 \\
Ge6 & 0.72 & Ge13 & 0.59 \\
Ge7 & 0.45 & & \\
\hline
\end{tabular}

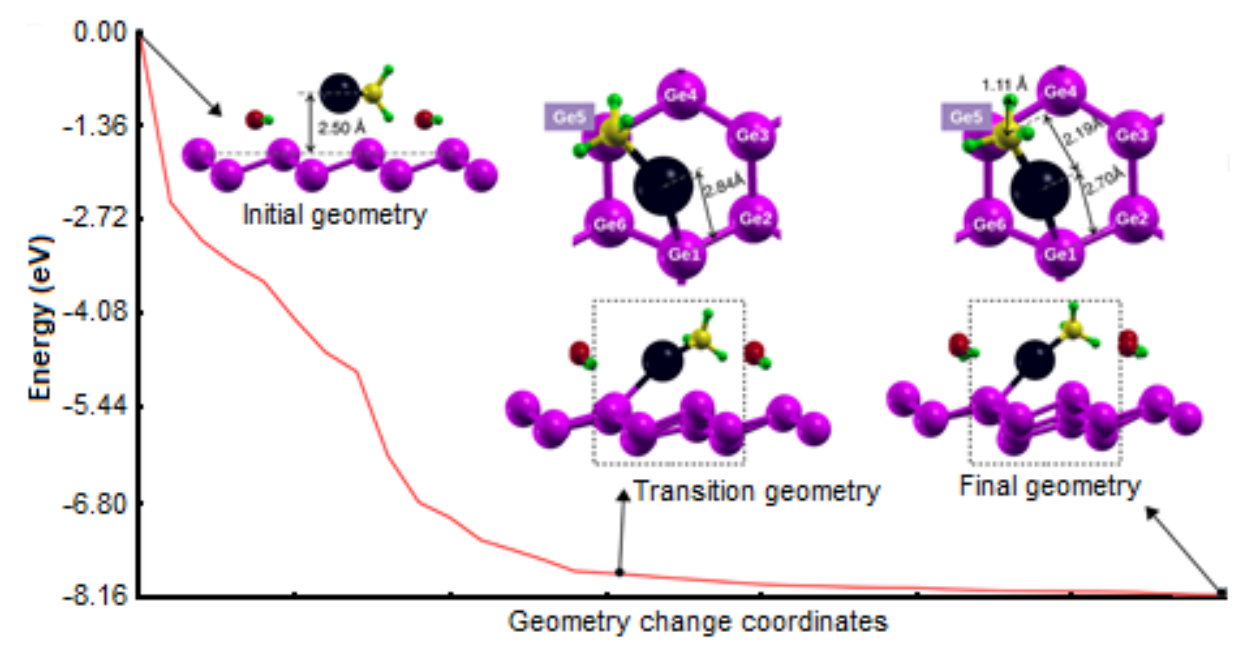

Fig 6. Atomic path of $\mathrm{MeHg} / \mathrm{G}$ system, the initial, transition, and final geometries are shown. The areas inside the dashed rectangular are also illustrated in the top view for clarity. The zero level is referenced to that the energy of initial geometry 


\section{- REFERENCES}

[1] Liu, G., Cai, Y., O’Driscoll, N., Feng, X., and Jiang, G., 2011, "Overview of Mercury in the Environment" in Environmental Chemistry and Toxicology of Mercury, Eds. Liu, G., Cai, Y., and O’Driscoll, N., Wiley, New Jersey, 1-12.

[2] Fitzgerald, W.F., and Lamborg, C.H., 2007, Geochemistry of Mercury in the Environment, Treatise Geochem., 9, 1-47.

[3] Gasong, B.T., Abrian, S., and Setyabudi, F.M.C.S., 2017, Methylmercury biosorption activity by methylmercury-resistant lactic acid bacteria isolated from West Sekotong, Indonesia, HAYATI J. Biosci., 24 (4), 182-186.

[4] National Research Council, 2000, Toxicological Effects of Methylmercury, National Academy Press, Washington, DC.

[5] Wang, J., Feng, X., Anderson, C.W.N., Xing, Y., and Shang, L., 2012, Remediation of mercury contaminated sites - A review, J. Hazard. Mater., 221-222, 1-18.

[6] Acun, A., Zhang, L., Bampoulis, P., Farmanbar, M., van Houselt, A., Rudenko, A.N., Lingenfelder, M., Brocks, G., Poelsema, B., Katsnelson, M.I., and Zandvliet, H.J.W., 2015, Germanene: The germanium analogue of graphene, J. Phys.: Condens. Matter, 27, 443002.

[7] Dávila, M.E., Xian, L., Cahangirov, S., Rubio, A., and Le Lay, G., 2014, Germanene: A novel twodimensional germanium allotrope akin to graphene and silicene, New J. Phys., 16, 095002.

[8] Li, L., Lu, S.Z., Pan, J., Qin, Z., Wang, Y.Q., Wang, Y., Cao, G.Y., Du, S., and Gao, H.J., 2014, Buckled germanene formation on $\mathrm{Pt}(111)$, Adv. Mater., 26 (28), 4820-4824.

[9] Derivaz, M., Dentel, D., Stephan, R., Hanf, M.C., Mehdaoui, A., Sonnet, P., and Pirri, C., 2015, Continuous germanene layer on $\mathrm{Al}(111)$, Nano Lett., 15 (4), 2510-2516.

[10] Kaloni, T.P., 2014, Tuning the structural, electronic, and magnetic properties of germanene by the adsorption of $3 \mathrm{~d}$ transition metal atoms, J. Phys. Chem. C, 118 (43), 25200-25208.
[11] Pang, Q., Zhang, C.L., Li, L., Fu, Z.Q., Wei, X.M., and Song, Y.L., 2014, Adsorption of alkali metal atoms on germanene: A first-principles study, Appl. Surf. Sci., 314, 15-20.

[12] Ye, M., Quhe, R., Zheng, J., Ni, Z., Wang, Y., Yuan, Y., Tse, G., Shi, J., Gao, Z., and Lu, J., 2014, Tunable band gap in germanene by surface adsorption, Physica E, 59, 60-65.

[13] Xia, W., Hu, W., Li, Z., and Yang, J., 2014, A firstprinciples study of gas adsorption on germanene, Phys. Chem. Chem. Phys., 16 (41), 22495-22498.

[14] Al Fauzan, M.R., Astuti, W.D., Al Fauzan, G., and Sholihun, 2018, The interaction of air pollutant molecules with germanene and silicene: A density functional theory study, Molekul, 13 (1), 92-97.

[15] Liu, G., Liu, S.B., Xu, B., Ouyang, C.Y., and Song, H.Y., 2015, First-principles study of the stability of free-standing germanene in oxygen atmosphere, $J$. Appl. Phys., 118, 124303.

[16] Ozaki, T., Kino, H., Yu, J., Han, M.J., Ohfuchi, M., Ishii, F., Sawada, K., Kubota, Y., Mizuta, Y.P., Ohwaki, T., Duy, T.V.T., Weng, H., Shiihara, Y., Toyoda, M., Okuno, Y., Perez, R., Bell, P.P., Ellner, M., Xiao, Y., Ito, A.M., Kawamura, M., Yoshimi, K., Lee, C.C., and Terakura, K., 2009, User's manual of OPENMX v.3.8., http://www.openmxsquare.org.

[17] Ozaki, T., 2003, Variationally optimized atomic orbitals for large-scale electronic structures, Phys. Rev. B, 67, 155108.

[18] Ozaki, T., and Kino, H., 2004, Numerical atomic basis orbitals from $\mathrm{H}$ to Kr, Phys. Rev. B, 69, 195113.

[19] Troullier, N., and Martins, J.L., 1991, Efficient pseudopotentials for plane-wave calculations, Phys. Rev. B, 43, 1993.

[20] Perdew, J.P., Burke, K., and Ernzerhof, M., 1996, Generalized gradient approximation made simple, Phys. Rev. Lett., 77, 3865.

[21] Zhuravlev, K.K., 2007, PbSe vs. CdSe: Thermodynamic properties and pressure dependence of the band gap, Physica B, 394 (1), 1-7.

[22] Murnaghan, F.D., 1944, The compressibility of media under extreme pressures, Proc. Natl. Acad. Sci. U.S.A., 30 (9), 244-247. 
[23] Birch, F., 1947, Finite elastic strain of cubic crystals, Phys. Rev., 71 (11), 809-824.

[24] Amalia, W., Nurwantoro, P., and Sholihun, 2019, Density-functional-theory calculations of structural and electronic properties of vacancies in monolayer hexagonal boron nitride (h-BN), Comput. Condens. Matter, 18, e00354.

[25] Hastuti, D.P., Nurwantoro, P., and Sholihun, 2019, Stability study of germanene vacancies: The firstprinciples calculations, Mater. Today Commun., 19, 459-463.

[26] Sholihun, Amalia, W., Hastuti, D.P., Nurwantoro, P., Nugraheni, A.D., and Budhi, R.H.S., 2019, Magic vacancy-numbers in h-BN multivacancies: The firstprinciples study, Mater. Today Commun., 20, 100591.

[27] Śpiewak, J.P., Vanhellemont, J., and Kurzydlowski, K.J., 2011, Improved calculation of vacancy properties in Ge using the Heyd-Scuseria-Ernzerhof range-separated hybrid functional, J. Appl. Phys., 110, 063534.

[28] Corsetti, F., and Mostofi, A.A., 2011, System-size convergence of point defect properties: The case of the silicon vacancy, Phys. Rev. B, 84, 035209.

[29] Probert, M.I.J., and Payne, M.C., 2003, Improving the convergence of defect calculations in supercells: An ab initio study of the neutral silicon vacancy, Phys. Rev. B, 67, 075204.

[30] Sholihun, Kadarisman, H.P., and Nurwantoro, P., 2018, Density-functional-theory calculations of formation energy of the nitrogen-doped diamond, Indones. J. Chem., 18 (4), 749-754.

[31] Al Fauzan, M.R., Astuti, W.D., Al Fauzan, G., and Sholihun, 2018, A first-principles investigation of the adsorption of $\mathrm{CO}$ and $\mathrm{NO}$ molecules on germanene, IOP Conf. Ser.: Mater. Sci. Eng., 367, 012051.

[32] Lee, K.W., and Lee, C.E., 2020, Strain and doping effects on the antiferromagnetism of AB-stacked bilayer silicene, Physica B, 577, 411816.

[33] Umam, K., Sholihun, Nurwantoro, P., Absor, M.A.U., Nugraheni, A.D., and Budhi, R.H.S., 2018, Biaxial strain effects on the electronic properties of silicene: The density-functional-theory-based calculations, J. Phys.: Conf. Ser., 1011, 012074.

[34] Scalise, E., Houssa, M., Pourtois, G., van den Broek, B., Afanas'ev, V., and Stesmans, A., 2013, Vibrational properties of silicene and germanene, Nano Res., 6 (1), 19-28. 